EESTI NSV TEADUSTE AKADEEMIA TOIMETISED. 28. KOIDE FOOSIKA * MATEMAATIKA. 1979, NR. 1

ИЗВЕСТИЯ АКАДЕМИИ НАУК ЭСТОНСКОИ ССР. ТОМ 28 ФИЗИКА * МАТЕМАТИКА. 1979, № 1

H. КЕЙС

удк 531.011

\title{
ОБ ЭКСТРЕМАЛЬНОМ СВОЙСТВЕ И РАЗДЕЛЕНИИ ДВИЖЕНИИ КАНОНИЧЕСКОЙ СИСТЕМЫ С ЛИНЕЙНЫМ ПО ГАМИЛЬТОНИАНУ И ИМПУЛЬСАМ ИНВАРИАНТОМ
}

I. KEIS. HAMILTONIAANI JA IMPULSSIDE LINEAARSE INVARIANDIGA KANOONILISE SUSTEEMI EKSTREMAALSEST OMADUSEST JA DEKOMPOSITSIOONIST

I. KEIS. ON EXTREMAL PROPERTIES AND DECOMPOSITION OF CANONICAL SYSTEM WITH HAMILTONIAN-IMPULSES LINEAR INVARIANT

\section{(Представлена Н. Алумяэ)}

1. Рассмотрим систему Лагранжа с непотенциальными силами при линейных идеальных неголономных связях $c_{\gamma k} q_{k}=c_{\gamma}$, либо голономную $\left(c_{\gamma k} \equiv c_{\gamma} \equiv 0\right)$ в случае $\operatorname{det}\left\|\partial^{2} L / \partial q_{j}{ }_{j} \partial q_{k}{ }_{k}\right\| \neq 0$ :

$$
\begin{gathered}
\left(p_{k}+\frac{\partial H}{\partial q_{k}}-Q_{k}\right) \delta q_{k}=0 \\
\left(H=p_{j} q_{j}^{*}-L, p_{j}=\frac{\partial L}{\partial q_{j}^{*}}, q_{k}=\frac{\partial H}{\partial p_{k}}, Q_{j}=Q_{j}(t, q, q), j, k=\overline{1, n}\right) .
\end{gathered}
$$

Система (1) имеет инвариант $G=p_{i} g_{i}\left(p_{n+1}=-H, \quad g_{i}=g_{i}(q, t)\right.$, $\left.c_{\gamma k}=c_{\gamma k}(q, t), c_{\gamma}=c_{\gamma}(q, t), f=d f / d t, i=\overline{1, n+1}\right)$ при условиях

$$
\begin{gathered}
\delta q_{k}^{1}=\varepsilon\left(g_{k}-g_{n+1} \dot{q_{k}}\right) \in\left\{\delta q_{k}\right\}, \\
Q_{k} \delta q_{k}^{1}=0, g_{n+1} \frac{\partial H}{\partial t}=g_{k}^{*} p_{k}-g_{k} \frac{\partial H}{\partial q_{k}}-H g_{n+1}^{.}(k=\overline{1, n}),
\end{gathered}
$$

где всюду предполагается достаточная гладкость используемых функций. Далее ограничимся вообще неавтономной голономной системой Гамильтона

$$
\begin{gathered}
\frac{d x}{d \tau}=[x, F], \frac{d p}{d \tau}=[p, F] \\
\left(F=H+p_{n+1}, t=x_{n+1}, x=\left(x_{i}\right)^{*}, p=\left(p_{s}\right)^{*}, i, s=\overline{1, n+1}\right), \\
g_{n+1} \frac{\partial H}{\partial t}+H\left(\frac{\partial g_{n+1}}{\partial t}+\frac{\partial g_{n+1}}{\partial q_{k}} \frac{\partial H}{\partial p_{k}}\right)=p_{k}\left(\frac{\partial g_{k}}{\partial t}+\frac{\partial g_{k}}{\partial q_{j}} \frac{\partial H}{\partial p_{j}}\right)-g_{j} \frac{\partial H}{\partial q_{j}} \\
(j, k=\overline{1, n)},
\end{gathered}
$$




$$
\begin{aligned}
& G=g_{i} p_{i}=g=\mathrm{const}\left(Q_{k}=0, G=[G, F]=\right. \\
& \left.=\frac{\partial G}{\partial x_{i}} \frac{\partial F}{\partial p_{i}}-\frac{\partial G}{\partial p_{s}} \frac{\partial F}{\partial x_{s}}=0, \quad F=-p_{0}=\text { const }\right),
\end{aligned}
$$

обладающей инвариантом $G$ в силу уравнения (2) для $H$. Расширенным обратимым точечным преобразованием $x_{i}^{\prime}=\partial T / \partial p_{i}^{\prime}, \quad F^{\prime}=F, \quad p_{i}=$ $=\partial T / \partial x_{i}\left(T=f_{i}(x) p_{i}^{\prime}, p_{i}^{\prime} x_{i}^{\prime *}=p_{i} x_{i}, \cdot i=\overline{1, n+1}\right)$ находим для (3) выражения

$$
\begin{gathered}
x_{i}^{\prime}=\partial F^{\prime} / \partial p_{i}^{\prime}, p_{i}^{\prime}=-\partial F^{\prime} / \partial x_{i}^{\prime}, x_{i}^{\prime}=f_{i}(x), p_{i}=p_{s}^{\prime} \partial f_{s} / \partial x_{i}, \\
p_{n+1}^{\prime}=G\left(x\left(x^{\prime}\right), p\left(x^{\prime}, p^{\prime}\right)\right)=g=\mathrm{const}, \partial F^{\prime} / \partial x_{n+1}^{\prime}=0 \\
\left(x^{\prime}=\left(x_{i}^{\prime}\right)^{*}, p^{\prime}=\left(p_{s}^{\prime}\right)^{*}, s=\overline{1, n+1)},\right.
\end{gathered}
$$

$F^{\prime}=F\left(x\left(x^{\prime}\right), p\left(x^{\prime}, p^{\prime}\right)\right), F(x, p)=F^{\prime}\left(x^{\prime}(x), p^{\prime}(x, p)\right)\left(F^{\prime}=F=-p_{0}=\right.$ const $)$, где $f_{k}(x), f_{n+1}(x)$ - функционально независимые решения уравнений

$$
\begin{gathered}
g_{i} \partial f_{k} / \partial x_{i}=0, g_{i} \partial f_{n+1} / \partial x_{i}=1 \\
\left(a_{v} b_{v}=\sum_{v=1}^{m} a_{v} b_{v}, \quad v=\overline{1, m}, \quad i, s=\overline{1, n+1}, \quad k, j=\overline{1, n}\right) .
\end{gathered}
$$

2. Обозначим через $X\left(x_{k}, p_{r}, g, p_{0}\right)=-p_{n}^{\prime}$ решение уравнения $F^{\prime}=$ $=-p_{0}$ в области $D^{\prime}=\left\{x^{\prime}, p^{\prime} \mid \partial F^{\prime} / \partial p_{n}^{\prime} \neq 0\right\}, \quad$ где $x^{\prime}{ }_{n} \neq 0 \quad(r, \sigma=$ $=\overline{1, n-1})$. Из (4), следуя $\left[{ }^{1}\right]$, получим $2(n-1)$-мерную систему Гамильтона

$$
\frac{d x_{r}^{\prime}}{d x_{n}^{\prime}}=\frac{\partial K}{\partial p_{r}^{\prime}}, \frac{d p_{r}^{\prime}}{\partial x_{n}^{\prime}}=-\frac{\partial K}{\partial x_{r}^{\prime}}\left(\left.K \equiv X\right|_{p_{0}=0}, F=F^{\prime}=0 \sim p_{0}=0\right)
$$

и уравнения зависимости $x_{n}^{\prime}, x_{n+1}^{\prime}\left(x_{r}^{\prime}, p_{\sigma}^{\prime}\right)$ от времени

$$
\frac{d t}{d x_{n}^{\prime}}=\left.\frac{\partial X}{\partial p_{0}}\right|_{p_{0}=0}, \quad \frac{d x_{n+1}^{\prime}}{d x_{n}^{\prime}}=\frac{\partial K}{\partial g}\left(p_{n+1}^{\prime}=g=\text { const, } p_{0}=\text { const }\right) .
$$

В области $D^{0}=\left\{D^{\prime} \cap F^{\prime}=0 \cap \operatorname{det} A \neq 0\right\} \quad\left(A=\left\|\partial^{2} K / \partial p_{\sigma} \partial p_{r}\right\|\right)$ для (6) введем новую функцию $P\left(x_{j}^{\prime}, d x_{\cdot r}^{\prime} / d x^{\prime}{ }_{n}, g\right)$ Лагранжа

$$
\begin{gathered}
P=\left(p_{r} \frac{d x_{r}^{\prime}}{d x_{n}^{\prime}}-K\right)_{p_{r}=\psi_{r}}=\frac{d t}{d x^{\prime}}\left(L-p_{n+1}^{\prime} f_{n+1}\right) \\
\left(\psi_{r}=\psi_{r}\left(x^{\prime}, \frac{d x^{\prime}{ }_{\sigma}}{d x_{n}^{\prime}}, g\right), f=\frac{d f}{d t}\right) .
\end{gathered}
$$

Из (6), (7) при условиях $x_{n}^{\prime}\left[t_{\alpha}\right]=c_{n \alpha}=$ const, $x_{r}^{\prime}\left[t_{\alpha}\right]=c_{r \alpha}=$ const $(\alpha=1,2)$ имеем стационарность действия $W$ в форме Якоби на траектории $x_{r}^{\prime}\left(x_{n}^{\prime}\right)$ движения системы Эйлера-Лагранжа с характеристической функцией

$W=\int_{x_{n 1}}^{x_{n 2}^{\prime}} P\left(x_{j}^{\prime}, d x_{r}^{\prime} / d x_{n}^{\prime}, g\right) d x_{n}^{\prime}\left(g=\right.$ const, $\left.x_{j \alpha}^{\prime}=x_{j}^{\prime}\left[t_{\alpha}\right], j=\overline{1, n}, r=\overline{1, n-1}\right)$.

Используя независимую переменную $t$, исходные $q_{k}$ и соотношения (5), $(7)$, для системы (3) с инвариантом $G$ получим следующее обобщение 
принципа стационарного действия Мопертюи-Лагранжа. В классе движений $\{q[t]\} \in C_{2}$, удовлетворяющих условиям

$$
\begin{gathered}
G_{*}=\left(g_{k}-g_{n+1} q_{k}\right) \partial L / \partial q_{k}+g_{n+1} . L=g=\text { const }(j, k=\overline{1, n}), \\
f_{j}\left(q_{k \alpha}, t_{\alpha}\right)=c_{j \alpha}=\operatorname{const}\left(G=g_{j} p_{j}-g_{n+1} H, L=p_{j} q_{j}-H, \alpha=1,2\right),
\end{gathered}
$$

функционал

$$
W *=\int_{t_{1}}^{t_{2}} R_{*} d \tau=\left.g f_{n+1}(q, t)\right|_{t_{2}} ^{t_{1}}+\int_{t_{1}}^{t_{2}} L d \tau\left(R_{*}=L-G_{*} f_{n+1}, G_{*}=\left.G\right|_{p=\partial L / \partial q}\right)
$$

имеет стационарное значение на движении системы Эйлера-Лагранжа с инвариантом $G_{*}$. Действительно, для (3) при ограничениях (8) находим $\Delta W *=0$, где $\Delta-$ асинхронная вариация Гельдера $\delta-t-\Delta t d / d t$.

Гравитирующие и гиростатические механические системы $\left[{ }^{2,3}\right]$ являются частным видом динамических моделей (1), (3) с линейным по гамильтониану и импульсам инвариантом $G$.

\section{ЛИТЕРАТУ РА}

1. Whittaker, E. T., A treatise on the analytical dynamics of particles and rigid bodies, Cambridge, University Press, 1904.

2. J a c ob i, C. G., Vorlesungen über Dynamik, Berlin, Reimer, 1884.

3. К е й с И., Изв. АН ЭССР, Физ. Матем., 24, № 3, 277-284 (19́75).

Институт кибернетики Академии наук Эстонской ССР
Поступила в редакцию 30/VI 1978 